\title{
High cadence near-infrared transit timing observations of extrasolar planets
}

\author{
Claudio Cáceres ${ }^{1,2}$, Valentin D. Ivanov ${ }^{1}$, Dante Minniti ${ }^{2}$ \\ Dominique Naef ${ }^{1}$, Claudio Melo ${ }^{1}$, Elena Mason ${ }^{1}$, \\ Fernando Selman ${ }^{1}$ and Grzegorz Pietrzynsky ${ }^{3}$ \\ ${ }^{1}$ European Southern Observatory ${ }^{2}$ Pontificia Universidad Católica de Chile \\ ${ }^{3}$ Universidad de Concepción \\ email: ccaceres@eso.org
}

\begin{abstract}
Currently the only technique sensitive to Earth mass planets around nearby stars (that are too close for microlensing) is the monitoring of the transit time variations of the transiting extrasolar planets. We search for additional planets in the systems of the hot-Neptune GJ-436 b, and the hot-Jupiter XO-1 b, using high cadence observations in the $J$ and $K_{S}$ bands, with the SofI and ISAAC instruments from La Silla Paranal Observatory. New high-precision transit timing measurements were used to derive new ephemeris. No statistically significant timing deviations were detected. We demonstrate that the high cadence ground based nearinfrared observations are successful in constraining the mean transit time to $30 \mathrm{sec}$, and are a viable alternative to space missions.
\end{abstract}

\section{Description}

We present the first results from our timing study of individual transits of extrasolar planets with infrared detectors, using the FastPhot mode available in SofI@NTT, and ISAAC@VLT in La Silla - Paranal Observatory, which provides us with an unprecedented time resolution of $0.05-0.3 \mathrm{sec}$, and minimum "dead" time for readout $(0.1 \%)$, generating a series of data cubes, with the target and one reference star in each windowed frame. The analyzed planets were the hot-Jupiter XO-1 b, and the hot-Neptune GJ-436 b.

\section{Results}

Figure 1 shows a light curve of planet XO- $1 \mathrm{~b}$ with an exposure time of $0.08 \mathrm{sec}$. The total number of data points is $\sim 190,000$. In the best fitting model determination, the error calculation was developed with a Bootstrapping simulation which takes into account the presence of red noise in the data (Cáceres et al. 2009). The O-C diagram for the hotNeptune GJ-436 b is also shown. These observations were taken on 17 May 2007, with the SofI@NTT at La Silla, in poor weather conditions. About 25,000 data points were collected using an exposure time of $0.24 \mathrm{sec}$, and for the best fitting model, we select stellar parameters from Gillon et al. (2007), and planetary parameters from Torres et al. (2007).

An individual transit timing accuracy of $30 \mathrm{sec}$ is achieved. The data show some TTVs (Transit Time Variations) of up to 98 sec. However, these deviations are consistent with zero, within their respective uncertainties. Further observations with higher accuracy are necessary to better constrain the properties of these system and to address the question of whether they contains other planets.

The new ephemeris thus obtained are:

$$
\text { XO-1 b: }
$$$$
T_{C}=2453808.91682(13)+E \times 3.9415128(28) \mathrm{HJD}
$$ 

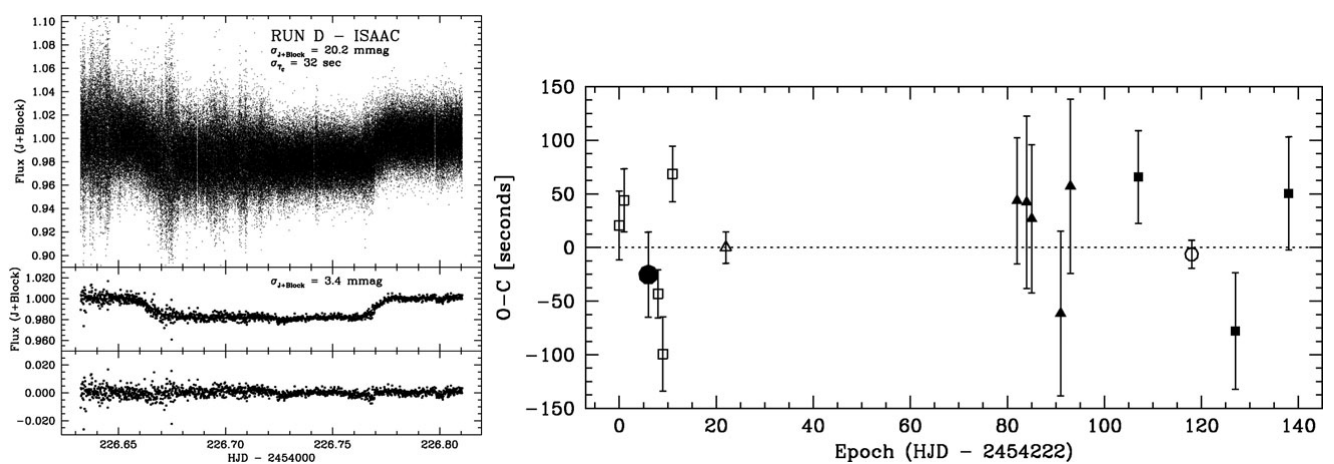

Figure 1. Left. A high cadence transit light curve for the transiting planet XO-1 b, taken with ISAAC@VLT the night of May 5, 2007. The best fitting model is shown, with planetary and stellar parameters taken from Holman et al. (2006) and McCullough et al. (2006) respectively. Right. The O-C diagram for our timing measurement of the transiting planet GJ 436b, and the data in the literature (see Cáceres et al. 2009 for more details).

$$
\text { GJ-436 b: } \quad T_{C}=2454222.61588(12)+E \times 2.6438986(16) \mathrm{HJD}
$$

\section{Conclusions}

We achieve transiting timing accuracies of about $30 \mathrm{sec}$ for individual transits. We find no significant evidence for perturbations of the orbital motion of GJ-436 b nor XO-1 b by other bodies in the system. Of course, a proper test of this hypothesis will require monitoring of multiple transits with the same or even higher accuracy. We demonstrate that the ground-based high-cadence observations of transiting extrasolar planets is an excellent technique for constraining the parameters of extrasolar planetary systems, because of the statistical significance of the obtained timing measurements. The timing precision is comparable with the space-based observations, making this method a good alternative to space missions, with their high cost and limited lifetime.

\section{Acknowledgements}

DM and CC are supported by the Basal Center for Astrophysics and Associated Technologies, and the FONDAP center for Astrophysics 15010003. This poster is also supported by Fondo ALMA-CONICYT 31070007, and the European Southern Observatory.

\section{References}

Cáceres, C., Ivanov, V. D., Minniti, D., Naef, D., Melo, C., Mason, E., Selman, F., \& Pietrzynski, G. 2009, $A \mathscr{E} A$, in press (arXiv:0905.1728)

Gillon, M., Pont, F., Demory, B.-O., Mallmann, F., Mayor, M., Mazeh, T., Queloz, D., Shporer, A., Udry, S., \& Vuissoz, C. 2007, A\&A 472, 13

Holman, M. J., Winn, J. N., Latham, D. W., O'Donovan, F. T., Charbonneau, D., Bakos, G. A., Esquerdo, G. A., Hergenrother, C., Everett, M. E., \& Pál, A. 2006, ApJ 652, 1715

McCullough, P. R., Stys, J. E., Valenti, J. A., Johns-Krull, C. M., Janes, K. A., Heasley, J. N., Bye, B. A., Dodd, C., Fleming, S. W., Pinnick, A., Bissinger, R., Gary, B. L., Howell, P. J., \& Vanmunster, T. 2006, ApJ 648, 1228 